\title{
A Comparison of the Differences Between Dutch and American Thyroid Association Guidelines for the Management of Pediatric Thyroid Cancer
}

\section{Catherine A. Dinauer}

Section of Pediatric Endocrinology, Department of Pediatrics, Yale School of Medicine, New Haven, Connecticut, U.S.A.

Review of: Lebbink CA, Dekker, BJ, Bocca G, Braat AJAT, Derikx JPM, Dierselhuis MP, de Keizer B, Kruijff S, Kwast ABG, van Nederveen FH, Nieveen van Dijkum EJM, Nievelstein RAJ, Peeters RP, Terwisscha van Scheltinga CEJ, Tissing WJE, van der Tuin K, Vriens MR, Zsiros J, van Trotsenburg ASP, Links TP, van Santen HM 2020 New national recommendations for the treatment of pediatric differentiated thyroid carcinoma in the Netherlands. Eur J Endocrinol 183:11-18. PMID: 32698145.

\section{SUMMARY}

\section{Background}

The American Thyroid Association (ATA) published their first set of guidelines for the care of children and adolescents with thyroid nodules and differentiated thyroid cancer (DTC) in 2015 (1). The document has been widely embraced, but areas of controversy remain, with ongoing debates regarding the role of molecular testing in evaluating pediatric thyroid nodules, the appropriate extent of initial surgery, and the use of postoperative radioactive iodine. Clinical practice varies across the globe, based on the interpretation of available data, local regulations, and available resources. Given differences in practice, members of professional societies outside the United States have embarked on developing their own pediatric thyroid cancer guidelines, including a Polish panel in 2016 (2) and, most recently, a panel from the Netherlands (3). The newest Dutch guidelines offer recommendations that overlap with and differ from the ATA guidelines (1) and are intended to guide current practice in the Netherlands and serve as the basis for developing formal guidelines for all European children.

\section{Methods}

A multidisciplinary committee that included the spectrum of specialties involved in thyroid cancer care as well as a patient representative was assembled. The panel used an adaptive approach in developing their guidelines rather than producing a de novo document. They conducted a PubMed literature search of existing recommendations and review articles, capturing studies on the management of DTC in patients $<18$ years of age published after 2000 and written in English or Dutch. The panel narrowed their results to six publications and scored the quality of each using the Appraisal of Guidelines, Research, and Evaluation (AGREE) method (4). Of the six publications, studied the ATA guidelines were determined to be the highest quality and were used as the springboard for the Dutch team's guidelines. The panel identified "discussion points" in the ATA document and addressed these through additional literature searches and consideration of practices and their expert opinions. 


\section{Results}

The complete guidelines were published as an Appendix to the article (3) and are also available online at www.oncoguide.nl. Many recommendations overlap with those of the ATA guidelines, which the authors specifically cite throughout. Like the ATA guidelines, the authors advocate that children with DTC be cared for by a centralized, multidisciplinary team and offer specifics on the minimum complement of specialists required, including a pediatric psychologist.

More overt differences in management emerge in the discussion of nodules. The panel outlines four "levels of care," stating that many children can initially be evaluated by a general pediatrician (level 2) with guidance from a pediatric endocrinologist as long as ultrasound can be done by an "experienced pediatric radiologist." The authors outline criteria for pursuing evaluation by a pediatric endocrinologist at a level 3 or 4 center, based on risk factors, age, and/or suspicious ultrasound features. Follow-up imaging is recommended for all nodules classified as Bethesda cytopathology category I, II, III, or IV by fine-needle aspiration biopsy (FNAB) (5); repeat FNAB is recommended for nodules initially classified as category I, III, or IV. Diagnostic lobectomy for indeterminate category III or IV nodules is not universally recommended but instead is selectively advised on the basis of suspicious ultrasound features, size $>4$ $\mathrm{cm}$, compressive symptoms, or family/patient preference. The authors recommend testing for BRAF mutation in Bethesda category $V$ (suspicious for malignancy) nodules, with consideration for testing for other specific genetic drivers (e.g., RET/PTC rearrangements, NTRK fusions) if BRAF is negative.

In terms of surgery, the Dutch panel "strongly recommends" total thyroidectomy for all children with malignant cytology, with consideration, by a multi- disciplinary tumor board, for less extensive surgery in those with microcarcinoma $(<1 \mathrm{~cm})$ and no signs of cervical lymph node involvement. For Bethesda category $\mathrm{V}$ nodules with a BRAF mutation, total thyroidectomy is recommended, while diagnostic hemithyroidectomy is advised if a different genetic variant is found or if there is no genetic variant identified. The authors agree with the ATA recommendation that suspicious lateral cervical lymph nodes should be evaluated by FNAB preoperatively, to determine whether therapeutic lateral neck dissection is indicated. However, they go on to state that lateral neck dissection may be undertaken without FNAB confirmation if a lateral cervical lymph node is deemed pathologic on palpation.

Postoperative use of ${ }^{131} \mathrm{I}$ is advised for all patients except those with microcarcinoma and disease confined to the thyroid. A pretreatment ${ }^{123}$ | wholebody scan is not recommended for staging. Citing expert opinion, the guidelines outline specific ${ }^{131}$ | activities to administer, based on extent of disease, initial surgery, and patient weight and pubertal stage.

The Dutch recommendations for follow-up surveillance and TSH suppression after initial treatment are similar to those in the ATA guidelines. One exception is that the Dutch guidelines do not advise consideration for follow-up nuclear medicine whole-body scan in patients classified as ATA Pediatric Intermediate-Risk or High Risk, and instead rely on the use of ultrasound and thyroglobulin measurement. The authors propose a role for FDG-PET imaging for patients with a detectable serum thyroglobulin (Tg) level but a negative ultrasound in determining whether additional ${ }^{131}$ I should be administered.

For patients with distant metastatic disease, the panel did not offer specific management recommendations and counsels referring to the ATA guidelines. 


\section{Conclusions}

The Dutch guidelines for the care of children with DTC provide management recommendations adapted from the 2015 ATA guidelines. Differences in approach include the limited use of molecular testing in select nodules, repeat imaging and FNAB of Bethesda categories III and IV nodules rather than diagnostic surgery, and a recommendation to administer adjuvant radioactive iodine to most patients.

\section{COMMENTARY}

The current article (3) outlines recommendations for the management of thyroid nodules and thyroid cancer in Dutch children developed by experts from the Netherlands adapted from the ATA pediatric thyroid nodules and thyroid cancer guidelines (1).

The Dutch approach to indeterminate nodules (Bethesda categories III and IV) is generally one of surveillance, while in the United States the practice is often to proceed with diagnostic lobectomy given that the rates of malignancy are higher for children than for adults (6). At the time the ATA guidelines were published in 2015, there were insufficient data to advise use of molecular testing in the evaluation of pediatric nodules (1). More data are now available. The Dutch guidelines recommend genetic evaluation solely for Bethesda category $\mathrm{V}$ nodules. Recent publications suggest that molecular testing can inform the management approach to each of the three indeterminate categories (Bethesda III, IV, and V) (6). The next iteration of the ATA pediatric thyroid cancer guidelines will need to address the evolving practice of genetic testing of nodules.

A glaring difference between the Dutch and the ATA guidelines is that the former advises broad use of radioactive iodine, while the latter advises more selective use. The European group cites the higher prevalence of lymph node involvement and distant metastases in children with DTC and their aim to minimize disease recurrence, whereas the
ATA guidelines argue for minimizing morbidity of treatment. More data are needed in order to refine the management guidance by both groups.

A limitation of the Dutch guidelines is that there is minimal citation of the data used in developing their recommendations. For example, the recommendation to proceed with lateral neck dissection based on palpation of adenopathy is not substantiated with a reference, leading one to wonder whether unnecessary surgeries will be done and morbidity increased. In addition, it is not clear why they advocate the use of FDG-PET imaging rather than a ${ }^{123}$ I whole body scan with or without a chest CT scan for children who are Tg-positive but have a negative ultrasound. The data on FDG-PET are almost exclusively from adults (in whom dedifferentiated disease is more likely to develop) and there are no studies showing utility in pediatric DTC. It is well known that there is a paucity of data on childhood thyroid cancer and thus there is high reliance on expert opinion. That said, the process of developing guidelines is becoming increasingly rigorous in the United States, with, for example, the adoption of the GRADE system (7) by many organizations. The level of detail explaining the rationale for each recommendation in the ATA pediatric guidelines (1) exceeds that in the Polish (2) and Dutch (3) documents. However, both European documents offer recommendations, often based on expert opinion, that will need to be considered by the ATA committee currently endeavoring to revise 
PEDIATRIC THYROID CANCER A Comparison of the Differences Catherine A. Dinauer Between Dutch and American Thyroid Association Guidelines for the Management of Pediatric Thyroid Cancer

the inaugural guidelines, using more recent data when appropriate.

The Dutch panel intends to update their recommendations at least every 3 years. In addition to traditional journal publication, they posted their guidelines on the Web as a "dynamic flowchart," which will allow for interim updates based on new evidence. This approach seems nimble and in step with using technology to rapidly provide the most current thinking, but it also raises the question of rigor in evaluating emerging data and revising recommendations intended to promote optimal long-term outcomes.

\section{References}

1. Francis GL, Waguespack SG, Bauer AJ, Angelos P, Benvenga S, Cerutti JM, Dinauer CA, Hamilton J, Hay ID, Luster M, et al. 2015 American Thyroid Association Guidelines Task Force. Management guidelines for children with thyroid nodules and differentiated thyroid cancer. Thyroid 25:716-759.

2. Niedziela M, Handkiewicz-Junak D, Małecka-Tendera E, Czarniecka A, Dedecjus M, Lange D, Kucharska A, Gawlik A, Pomorski L, Włoch J, et al. 2016 Diagnostics and treatment of differentiated thyroid carcinoma in children-Guidelines of Polish national societies. Endokrynologia Polska 67:628-642.

3. Lebbink CA, Dekker, BJ, Bocca G, Braat AJAT, Derikx JPM, Dierselhuis MP, de Keizer B, Kruijff S, Kwast ABG, van Nederveen FH, et al. 2020 New national recommendations for the treatment of pediatric differentiated thyroid carcinoma in the Netherlands. Eur J Endocrinol 183:11-18.
4. Brouwers M, Kho ME, Browman GP, Cluzeau F, Feder G, Fervers B, Hanna S, Makarski J, AGREE Next Steps Consortium. 2010 AGREE II: Advancing guideline development, reporting and evaluation in health care. CMAJ 182:E839-E842.

5. Cibas ES, Ali SZ. 2017 The 2017 Bethesda system for reporting thyroid cytopathology. J Am Soc Cytopathol 6:217-222.

6. Bauer AJ. 2019 Thyroid nodules in children and adolescents. Curr Opin Endocrinol Diabetes Obes 26:266-274.

7. Guyatt GH, Oxman AD, Vist GE, Kunz R, FalckYtter Y, Alonso-Coello P, Schünemann HJ, GRADE Working Group 2008 GRADE: An emerging consensus on rating quality of evidence and strength of recommendations. BMJ 336:924-926. 